Key points

- Asthma is a complex heterogeneous disease that likely comprises several distinct disease phenotypes.

- Phenotypes can be classified by analysing single or multiple manifestations of disease including symptoms and clinical measurements using various clustering approaches.

- Phenotypes classified in this way show differences in associations with clinical outcomes and with underpinning genetic pathways.

- Big data will add to the complex multidimensionality of these approaches but the challenge is to identify interventions that are specific to one or more of these phenotypes to realise the potential of personalised medicine. 


$\begin{array}{lll}\sum_{\text {A. John Henderson }} & \begin{array}{l}\text { School of Social and } \\ \text { Community Medicine, } \\ \begin{array}{l}\text { University of Bristol, } \\ \text { Bristol, UK }\end{array}\end{array} & \begin{array}{l}\text { University of Bristol, School } \\ \text { of Social and Community } \\ \text { Medicine, Oakfield House, }\end{array} \\ & \begin{array}{l}\text { Oakfield Grove, Bristol, } \\ \text { BS8 2BN, UK }\end{array}\end{array}$

\section{Childhood asthma phenotypes in the twenty-first century}

\section{Summary}

A substantial proportion of asthma has its origins in childhood but attempts to identify modifiable exposures that trigger asthma inception have yielded disappointing results. It is increasingly recognised that asthma may not be a single disease entity but instead consists of a number of phenotypes that share common features perhaps with different aetiologies and pathophysiological pathways leading to disease.

The aim of this review is to describe approaches to defining asthma phenotypes on the basis of clinical and epidemiological data and to consider the clinical implications of the phenotypes described. Differences in clinical features (atopy, lung function, prognosis) have been associated with phenotypes derived from unsupervised statistical approaches to phenotype definitions. Greater understanding of the genetic pathways underpinning asthma has also led to some insights into asthma endotypes that manifest as different clinical phenotypes. However, the challenge to translate these findings into interventions to change the natural history of asthma has yet to be overcome. Technological advances are driving the creation of massive data resources that can be brought to bear on this problem. Whether they will finally solve the problem of phenotypic complexity in asthma remains to be discovered. If they do, the possibility of personalised interventions for asthma may eventually be realised.

\section{Introduction}

Asthma has been recognised for a very long time: the first clinical description of the disease is generally ascribed to Aretaeus of Cappadocia, who practised in Rome and Alexandria in the second century AD. Although the concept of asthma has changed over time, it has tended to have been
Statement of Interest None declared. 
regarded as a single disease with variations in clinical course and outcome. Now, almost two millennia after its first published description, it is widely believed that the clinical variability of asthma signifies not one disease but many, united by some common clinical features, such as intermittent wheezing and reversible airways obstruction. This has given rise to the concept of different phenotypes of asthma, each of which may arise from different pathological processes (endotypes) and which may respond differently to asthma treatments.

The term "phenotype" can be used to describe any observable properties or traits of an organism. These are generally regarded to arise from interactions between the genotype and environmental influences. A disease phenotype is described by clinical characteristics, which can include biochemical and other measured variables as well as physical features, without reference to an underlying pathophysiological process. The term "endotype" is used to describe a disease sub-type based on distinct pathological mechanisms. Therefore, a clinical phenotype of asthma may be underpinned by a number of discrete endotypes, each of which leads to a final common pathway of disease manifestations that characterise that particular phenotype [1]. Conversely, a discrete endotype, for example, one classified by a particular inflammatory pathway such as eosinophilic airway inflammation, could be extant in a number of different clinical phenotypes.

The importance of disentangling the various phenotypes and endotypes of asthma is two-fold: asthma prevention and treatment. A greater understanding of the aetiopathological mechanisms underpinning different disease phenotypes could reveal modifiable factors that initiate disease or influence its natural history enabling development of primary or secondary prevention strategies. Alternatively, drug-targetable pathways, applicable only in certain sub-types of asthma, could be discovered, opening the way for personalised medicine in asthma treatment. The interest in asthma phenotypes is exemplified by the explosion in publications on this topic since the early 1990s, before which there were generally fewer than 10 papers per year, increasing to over 300 per year in 2012 and 2013 (using PubMed, www.ncbi.nlm.nih. gov/pubmed). In this article, I will review the developing concepts of asthma phenotypes in
Big data

A massive volume of both structured and unstructured data that is so large that it is difficult to process using traditional database and software techniques.

children which have arisen from a variety of different approaches to their classification, consider the implications for disease aetiology and treatment, and suggest new possibilities in approaching this problem in the era of "big data".

\section{Asthma phenotypes based on symptoms}

It has long been recognised that different patterns of asthma symptoms in childhood may be associated with variations in the natural history of the disease. Henry Blair, a London-based general practitioner reported a 20-year follow up of children presenting with asthma, noting an association between symptom severity, but not age of onset, and persistence of asthma into adulthood. The Melbourne Epidemiological Study of Childhood Asthma found similar relationships in children with wheezing and severe asthma recruited at 7 and 10 years of age respectively and followed to mid-adulthood (42 years). Those who had mild "wheezy bronchitis" at age 7 years were less likely to report persistent or frequent asthma than those with severe asthma in childhood; in this group, over $75 \%$ continued to report persistent or frequent asthma in adult life. Further evolution of the concept that early childhood symptom pattern was associated with disease natural history was triggered by a seminal paper reporting on an unselected birth cohort in Tucson, AZ, USA. MARTINEZ et al. [2] categorised wheezing during the first six years of childhood into three distinct patterns on the basis of presence or absence of wheezing before the age of 3 years and its persistence or incidence to age 6 years: transient early, persistent and late-onset wheeze. As the researchers had access to lung function measurement in infancy as well as at the later assessment, it was possible to relate trajectories of lung function with the three wheezing phenotypes and to compare 
these with healthy, non-wheezing controls. This showed that infants with transient early wheeze had lower lung function (V'max,FRC) than controls shortly after birth, whereas those with persistent wheeze had lung function that was nearly equivalent to the controls in early infancy before symptoms developed but diverged to a substantially lower forced expiratory volume in $1 \mathrm{~S}$ (FEV $\left.\mathrm{FV}_{1}\right)$ compared with controls at age 6 years. They were also more likely to have a family history of asthma and to have higher serum immunoglobulin (Ig) E concentrations than both controls and transient early wheezers. Therefore, this classification seemed to segregate children with early onset of wheeze into those with transient symptoms, usually in response to viral triggers, in association with diminished airway function around the time of birth and those whose wheeze persisted in association with an atopic phenotype, akin to early onset asthma.

Of course, one of the problems of classifying wheezing illness according to its time course in this way is that the ability to recognise different phenotypes is retrospective. This approach has been enormously influential in seeking to determine the aetiological influences on asthma development and in further defining the natural history of asthma through the life-course. However, it is of limited or no utility in helping the clinician to differentiate early onset asthma from the majority of young children with wheeze who have a transient illness associated with probable abnormal intrauterine airway development. A European Respiratory Society task force attempted to resolve this dilemma by suggesting a dichotomy of early wheezing phenotypes based on recognised trigger factors: episodic viral wheeze, which occurred only in the presence of viral respiratory infections; and multiple-trigger wheeze, which occurred in response to both viral and other triggers [3]. Abnormal structural and/or functional development of the airways during intrauterine life would be associated with low lung function at birth and a tendency to wheeze in the context of airway inflammation induced by viral respiratory infections, resulting in an episodic pattern of wheezing that was likely to remit as the airways grew. Alternatively, multiple-trigger wheeze was more likely to persist between discrete episodes and to be associated with persistent symptoms, although evidence that this represented allergic airway inflammation was acknowledged as lacking. An expert working group recently reviewed these recommendations and considered them to be an oversimplification of the complex nature of wheezing illness in young children that varies over time in both the nature of symptoms and in the patterns of response to various environmental triggers. There remains a great deal of uncertainty about the optimal approach to therapy, even if it is indicated at all, in the majority of this population of infants with episodic or persistent wheezing.

Recently, a number of research groups have returned to the problem of classification of early wheezing into constituent phenotypes using unsupervised, data-driven approaches. Although these can be regarded as hypothesis-free, in that no pre-specified conditions are applied to the number or constitution of phenotypes, it has rightly been pointed out that studies to date have not been completely agnostic as they are dependent on selection of the input variables. Various statistical methods have been applied that largely rest on clustering of cases according to similarities of the characteristics considered; these have ranged from bivariate approaches (a single symptom (usually wheezing) reported over time) through more sophisticated, multivariable approaches that simultaneously model several variables, including symptoms and other clinical characteristics. Using principalcomponents analysis of multiple symptoms, SMITH et al. [4] in Manchester, UK, identified a five-component model (wheeze, wheeze with irritants, wheeze with allergens, cough, chest congestion) that explained a high proportion $(49.8 \%)$ of the variance of respiratory symptoms at age 5 years and was associated with measured lung function and with recognised asthma risk factors. Because different components were associated with differing hallmarks of asthma, such as lung function, airway reactivity, family history and quantitative allergic responses, the authors concluded there was evidence of differing underlying pathophysiology. This would imply that a unified approach to treating asthma is almost doomed to fail and that efforts should be directed towards identifying these biological underpinnings to enable development of more intelligent, targeted asthma therapy. SPYCHER et al. [5] applied latent class analysis, a multidimensional clustering technique, to 
data from the Leicester cohorts at around $3-6$ years of age, including respiratory symptoms, lung function, airway responsiveness and allergic sensitisation. These authors identified five phenotypes, three of which were characterised by wheezing: transient viral wheezing, atopic persistent wheeze and non-atopic persistent wheeze [5]. These showed some variation in their associations with lung function and airway responsiveness and in the prognosis of persistent asthma at later time points, with atopic persistent wheeze having the highest prevalence of ongoing symptoms.

We also used latent class analysis to derive early wheezing phenotypes based on repeat assessment of parent-reported wheezing in a large, longitudinal, population-based birth cohort (the Avon Longitudinal Study of Parents and Children (ALSPAC)). Using annual reports of wheezing to age 7 years in almost 6000 children, we reported five phenotypes defined by their temporal wheezing trajectory (fig. 1), including two that had not been previously recognised [6]. Interestingly, three of the phenotypes to emerge from this analysis mapped directly to those reported by MARTINEZ et al. [2] from the Tucson study: transient early wheeze, late onset wheeze and persistent wheeze. We also identified a prolonged early wheezing phenotype that we believe to be a severe form of transient early wheeze, as mid-expiratory flow measured at age 7 was lower but other associations with measured outcomes were similar to those for transient early wheeze.

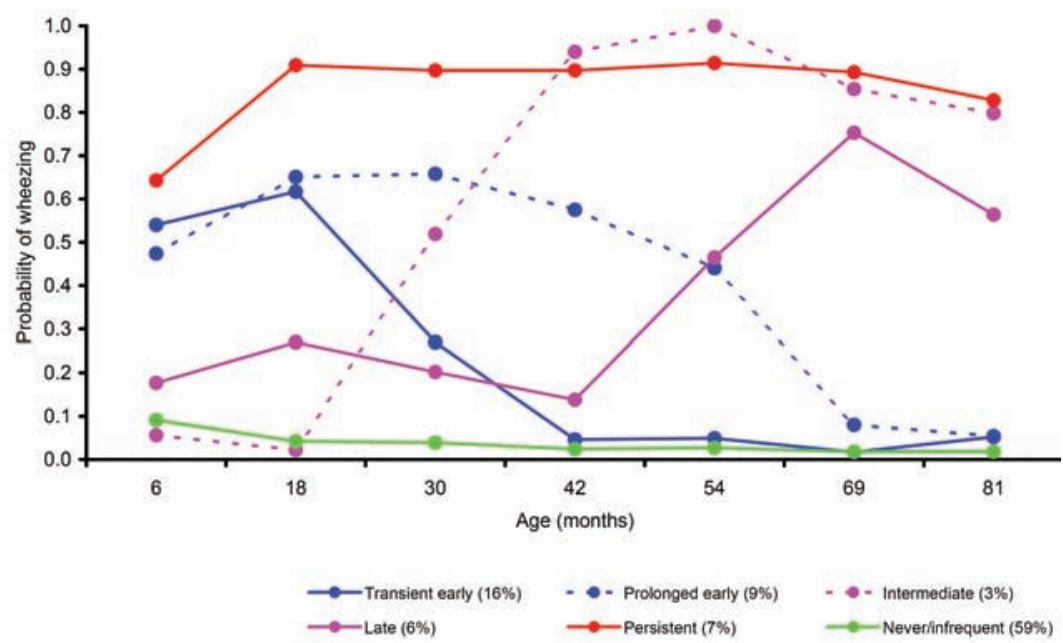

Figure 1

Asthma phenotypes defined over time. Reproduced from [6] with permission from the copyright holder.
The fifth phenotype, intermediate onset wheeze, was characterised by onset around 18 months of age and strong associations with asthma, allergic sensitisation and increased airway responsiveness in later childhood. These results have been confirmed in independent cohorts, including the Dutch Prevention of Asthma and Mite Allergy (PIAMA) study [7], Southampton Women's Survey (SWS) [8] and the Protection against Allergy Study in Rural Environments (PASTURE) [9]. In the last of these, the authors compared latent class phenotypes from birth to age 6 years with phenotypes derived from conventional clinical approaches using atopy, clinical measures (lung function and fractional exhaled nitric oxide $(\mathrm{FeNO}))$ and medication use. There was a high correlation of two clinical phenotypes with the latent class analyses: multi-trigger wheeze and recurrent unremitting wheeze. The latter was particularly important as a high proportion of children in this group did not have an asthma diagnosis and was not treated with asthma medication despite having evidence of respiratory morbidity, lung function deficits and poor response to inhaled short-acting bronchodilators. It is also evident from trajectories presented in this paper that, in contrast to latent class phenotypes, the clinical phenotypes did not diverge before the age of 2 years. This would be consistent with clinical experience that asthma diagnosis is difficult in the preschool years. The remarkable congruence between clinical and latent class phenotypes in this study has verified the utility of the latter approach to revealing novel phenotypes of wheezing illness and asthma in young children. However, attempts to identify different aetiopathological influences of individual phenotypes have been disappointing to date, with confirmation of existing knowledge that breast feeding and tobacco smoke exposure were respectively negatively and positively associated with transient and prolonged early wheeze [10].

It is important to note that latent classes are just that: latent. They provide a useful tool to examine latent structures of data within a sample but do not in themselves have any clinical utility. An individual subject can be assigned a relative probability of belonging to several different classes but is not a member of any one of these (it is rare to have a probability of 1.0 for membership of one class and therefore zero for all the others) so cannot be classified as having a specific 
phenotype. However, this approach has led to the recognition of phenotypes that are helping to uncover clinically relevant subtypes of asthma. The endotypes underpinning these have yet to be discovered but could potentially lead to new targets for asthma prevention and treatment.

\section{Asthma phenotypes based on clinical characteristics}

One of the challenges of classifying clinical phenotypes of asthma in children arises from the practical and ethical difficulties of obtaining biological material from the disease endorgan, the paediatric airway. Identification of the inflammatory infiltrate in airway samples has helped in the classification of adult asthma phenotypes using material from bronchial biopsies and induced sputum samples. These techniques have been applied to selected populations of young children, the former usually being restricted to those in whom there is a clear clinical indication and expectation of benefit. Thus, to date, they have tended to be restricted to studies of the most severely affected individuals or to opportunistic samples; for instance, those having elective surgery for some other problem. In the latter group, both bronchoscopic and non-bronchoscopic approaches have been used to obtain airway samples, including bronchial brushings to obtain airway epithelial cells for culture. SHIELDS et al. [11], in Belfast, have utilised non-bronchoscopic techniques in routine surgical patients to classify airway inflammation in viralassociated wheeze and atopic asthma in children, reporting increased esosinophils and mast cells in the airway of children with atopic asthma but not with viral-associated wheeze or atopy alone. DE BLIC et al. [12] retrospectively analysed clinically indicated bronchoscopies in children younger than 3 years and reported that those with severe, recurrent wheezing had evidence of neutrophil-predominant airway inflammation, an inflammatory endotype that is classically associated with COPD in adults; however, it is also reported in severe and non-steroid responsive asthma. There is clearly overlap between this endotype and preschool wheeze with eosinophilic airway inflammation and airway wall remodelling, as described by SAGLANI et al. [13].
A phenotype of particular interest in children has been severe and difficult-tocontrol asthma, largely due to its disproportionate morbidity burden and the challenges of treating this form of asthma with a limited choice of therapeutic options. In this group, there has been clear clinical justification for including invasive investigations in the diagnostic pathway, including bronchoscopy and endobronchial biopsy. BusH et al. [14] and his group have reported evidence for phenotypic variation in this group of children on the basis of exhaled nitric oxide (NO) measurements and responses to systemic corticosteroids; a steroid insensitive phenotype with persistent symptoms and raised $\mathrm{NO}$ and a phenotype with low exhaled NO and persistent symptoms. On the basis of their and others' experience with endobronchial biopsy findings, they have described inflammatory cell infiltration, respiratory basement membrane changes and cytokine expression, leading to a suggestion that phenotype-specific treatment may be feasible in this population [15]. Of note, many children in this category but by no means all had eosinophilic airway inflammation with thickening of the basement membrane.

Given the limitations of administering bronchoscopy to the majority of children with asthma to identify the inflammatory endotype, other methods have been sought including the use of induced sputum and biomarkers in exhaled breath. Induced sputum sampling has been used to recover lower airway samples in cystic fibrosis or for the diagnosis of tuberculosis in infants aged only a few months old. Although this method appears to be a promising addition to the diagnostic armamentarium that can be applied in population-based studies as well as clinical samples, concerns have been raised about the stability of the inflammatory endotypes in children with both severe and mild-to-moderate asthma [16]. Using endotypes derived from classification of adult asthma into eosinophilic and non-eosinophilic forms, FLeming et al. [16] reported that a high proportion $(41 \%)$ of children with asthma fulfilled criteria for eosinophilic asthma on one occasion and non-eosinophilic on another, without concomitant changes in treatment or exhaled NO measurements. This uncertainty may limit the utility of sputum induction as a method to aid classification of endotypes in population samples of children with wheezing, 


\section{Educational questions}

1. In the ERS defin-

itions, episodic viral wheezing is distinguished from multipletrigger wheezing in young children by which of the following characteristics?

a. More likely to have a family history of asthma

b. A tendency to recurrence of wheeze in adolescence

c. Lower lung function in infancy that improves with time

d. Evidence of sensitisation to foods and aeroallergens

e. Evidence of allergic airway inflammation

2. In the PASTURE study, latent class analysis was used to define wheezing phenotypes. Which two of the following clinical phenotypes were most closely correlated with latent classes?

a. Recurrent unremitting wheeze

b. Asthma diagnosis

c. Frequent wheeze

d. Multiple-trigger wheeze

e. Episodic wheeze unless it is performed repeatedly and longitudinally.

An alternative approach to endotyping asthma is to use biomarkers in the breath, blood or urine as indicators of specific inflammatory processes in the airways. A number of these have been developed, including fractional exhaled $\mathrm{NO}(\mathrm{FeNO})$ and a variety of mediators of inflammation and oxidative stress in exhaled breath condensate. Serum eosinophilic cationic protein (ECP), urinary eicosanoids and, recently, periostin have been proposed as systemic biomarkers of T-helper (Th) 2 mediated airway inflammation. In the opinion of the author, none of these has been convincingly demonstrated to enhance phenotypic discrimination in children's asthma beyond their associations with allergic inflammation, which is clearly an important endotype but probably underpins more than one clinical phenotype.

Classically, asthma in children has been regarded as a Th-2 mediated disease that is strongly associated with sensitisation to allergens and other allergic disease manifestations, including eczema and allergic rhinitis. This has been conceptually encapsulated in the allergic march. However, many children with one allergic disease do not progress to another so there may be specific phenotypes of asthma with eczema or hay fever. Alternatively, a common pathway such as induction of allergic sensitisation through skin barrier defects, could promote the development of allergic airway inflammation; for example, children who are deficient in filagrin have a higher risk of asthma but only in the presence of eczema [17]. Atopy is certainly more strongly associated with persisting wheezing phenotypes than with transient viral-triggered wheezing and the classification of asthma into an atopic and non-atopic phenotype is relatively straightforward from a clinical perspective. However, this approach may be to fall into a trap of regarding atopy as a single disease entity analogous to the previous concept of asthma; several groups have now applied clustering methods to atopy and have reported discrete phenotypes that differ in their associations with asthma $[18,19]$. Therefore, it seems likely that multidimensional and sophisticated statistical approaches will be needed to untangle the component phenotypes of even seemingly straightforward clinical categories. Other clinical phenotypes (e.g., it has been suggested that a discrete obesity-asthma phenotype exists) could well be subject to the same complexities of association, which may explain some of the discrepancies that exist in the literature relating these two increasingly common phenomena.

\section{Lessons from genetic studies}

As noted above, the evidence that symptomdefined phenotypes of asthma are discrete pathophysiological entities rather than lying on a single spectrum of disease has yet to be supported by strong evidence that they are associated with different environmental risk factors. The exception is transient episodic viral wheezing, which may be nothing to do with asthma (as an airway inflammatory disease) but represents an airway developmental abnormality that is associated with prenatal exposure to tobacco smoke metabolites from maternal smoking. However, asthma almost certainly arises as a consequence of interaction between genetic predisposition and environmental exposures, perhaps at critical periods during the course of life. Therefore, differential associations of asthma risk variants with specific phenotypes would provide supportive evidence of biological differences between them. The first large-scale genome-wide association study (GWAS) of asthma reported a strong association between physician-diagnosed asthma and several single nucleotide polymorphisms (SNPs) in the region 17921 near the $O R M D L_{3} / G S D M B$ gene [20]. Interestingly, the association of SNPs at this locus with asthma appeared to be restricted to childhood as opposed to adult-onset asthma and there was no evidence of association with atopy (defined by total serum IgE). We have followed up some of these associations in relation to wheezing phenotypes defined by latent class analysis [6] and with other clinical features of asthma, such as lung function, allergic sensitisation and bronchial responsiveness. The results suggested specific association of the 17921 locus with persistent and intermediateonset wheeze and with bronchial responsiveness but not with atopy or lung function [21]. A further analysis of results from the same GWAS in which we included thousands of SNPs that did not reach the stringent false discovery threshold applied to agnostic GWAS analyses showed that the top SNPs from the GWAS 
predicted the symptom-based persistent and intermediate-onset wheeze more strongly than doctor-diagnosed asthma (from which the original association was derived) [22]. Additionally, SNPs below the genome-wide significance threshold were associated with bronchial responsiveness and atopy, suggesting some overlap between the genetic underpinning of these phenotypes with asthma.

Two other genome-wide approaches have been applied to studying genetic relationships between asthma phenotypes and endotypes; GWAS of specific clinical phenotypes of asthma and the application of results from GWAS of asthma endotypes. An example of the former was a GWAS of asthma with hay fever, which identified 11 SNPs associated with this phenotype; two of which had not been identified previously [23]. Although they were all associated with asthma and hay fever independently, the association was stronger for the combined phenotype, which is likely to be explained by the association of allergic asthma and hay fever with allergy-related genetic variants. Therefore, it is questionable if this takes our thinking about phenotypes much further forward than the well-described dichotomy between atopic and non-atopic asthma. There has been a long-running recognition of a link between asthma and obesity, with the suggestion that obesityrelated asthma may represent a distinct phenotype. MeLEN et al. [24] conducted a GWAS of BMI stratified for asthma and found a hint that there may be shared genetic origins of asthma and obesity, although they could not replicate this association in an independent population. Endotypes that have been studies in GWAS include exhaled nitric oxide [25], lung function [26] and allergic sensitisation [27]. These studies generally confirm that these endotypes have distinct genetic associations in addition to those that may be shared with asthma, such as SNPs in the 17921 region associated with FeNO. Our meta-analysis of GWAS of allergic sensitisation through the EAGLE (Early Genetics and Lifecourse Epidemiology) consortium discovered 10 novel SNPs in or near genes in biologically plausible pathways. Testing these SNPs in published GWAS of asthma showed evidence of association with directional consistency for nine of them, confirming that allergic pathways are involved in asthma. We have recently reported associations of COPDassociated genes with transient early wheeze and lung function in children, emphasising the characteristic associated lung developmental abnormality of this particular phenotype and also its potential implications for life course respiratory health. However, important though this observation is, it can be regarded as confirmatory evidence rather than adding new insights into early childhood phenotypes. It should be noted that the studies cited here represent a small fraction of asthma genetic research, which has pointed to novel pathways and targets associated with the disease. However, it is not yet clear whether the majority of these specific biological pathways (which are themselves endotypes) are manifested by different phenotypes. The challenge for personalised medicine will be to find phenotypic features that identify individuals that may be amenable to treatment or intervention with modifying agents for highly specific endotypes.

\section{What does the future hold?}

There will undoubtedly be more data, possibly more than we will know what to do with. Linkage to routine healthcare data is already well established in some countries and is being rapidly embraced by others, enabling access to information on diagnosis, severity, treatment and response to treatment. Genome-wide data is extensively available and will be complemented by next generation sequencing data. In addition, 'omics technologies will generate extensive data about a number of characteristics of relevance to asthma and its phenoytpes. These include the epigenome, the inflammasome, the metabolome, and the microbiome. Biomarkers of exposure are being sought to strengthen causal associations with clinical outcomes. These include using genes as instrumental variables in Mendelian randomisation studies, capturing biological changes in response to exposures through epigenomics and using multiple instruments to capture the totality and complexity of environmental exposures (exposomics) rather than consider them individually. Some of these approaches present challenges, including access to relevant tissues, in addition to the bioinformatics resources required to process and analyse the large datasets generated by these methodologies. Different approaches to analysis are already in
3. Genome-wide association studies have consistently identified single nucleotide polymorphisms in the 17921 region (near ORMDL3) as being strongly associated with asthma. Which two of the following phenotypes have also been associated with SNPs in this region?

a. Allergic sensitisation

b. Transient early wheezing

c. Fractional exhaled nitric oxide

d. $\mathrm{FEV}_{\mathrm{l}} / \mathrm{FVC}$ ratio

e. Intermediateonset wheezing

4. In the severe asthma phenotype in children, the most likely endotype identified in bronchial biopsy specimens is which of the following

a. Neutrophil predominant airway inflammation

b. Paucicellular infiltrate with extensive basement membrane thickening

c. Non-inflammatory airway smooth muscle hypertrophy

d. Eosinophilic inflammation with basement membrane thickening

e. Goblet cell hypertrophy with increased mucus secretion 


\section{Suggested} answers

1. $\mathrm{C}$

2. a, d

3. c, e

4. d development and application, including machine learning systems. The possibilities arising from these large, sophisticated and interdisciplinary approaches to understanding the inter-relationships of biological data are exciting and potentially limitless.
However, there is a need to translate the output of such systems to clinically relevant applications if we are to realise their potential to transform approaches to disease management according to disease stratification.

\section{References}

1. Agache I, Akdis C, Jutel M, et al. Untangling asthma phenotypes and endotypes. Allergy 2012; 67: 835-846.

2. Martinez FD, Wright AL, Taussig LM, et al. Asthma and wheezing in the first six years of life. $N$ Eng J Med 1995; 332: 133-138.

3. Brand PL, Baraldi E, Bisgaard $\mathrm{H}$, et al. Definition, assessment and treatment of wheezing disorders in preschool children: an evidence-based approach. Eur Respir J 2008; 32: 1096-1110.

4. Smith JA, Drake R, Simpson A, et al. Dimensions of respiratory symptoms in preschool children: population-based birth cohort study. Am J Respir Crit Care Med 2008; 177: 1358-1363.

5. Spycher BD, Silverman M, Brooke AM, et al Distinguishing phenotypes of childhood wheeze and cough using latent class analysis. Eur Respir J 2008; 31: 974-981.

6. Henderson J, Granell R, Heron J, et al. Associations of wheezing phenotypes in the first 6 years of life with atopy, lung function and airway responsiveness in mid-childhood. Thorax 2008; 63: 974-980.

7. Savenije OE, Granell R, Caudri D, et al. Comparison of childhood wheezing phenotypes in 2 birth cohorts: ALSPAC and PIAMA.J Allergy Clin Immunol 2011; 127: 1505-1512.

8. Collins SA, Pike KC, Inskip HM, et al. Validation of novel wheeze phenotypes using longitudinal airway function and atopic sensitization data in the first 6 years of life: evidence from the Southampton Women's survey. Pediatr Pulmonol 2013; 48: 683-692.

9. Depner M, Fuchs O, Genuneit J, et al. Clinical and epidemiologic phenotypes of childhood asthma. Am J Respir Crit Care Med 2014; 189: 129-138.

10. Granell R, Sterne JA, Henderson J. Associations of different phenotypes of wheezing illness in early childhood with environmental variables implicated in the aetiology of asthma. PLoS One 2012; 7: e48359.

11. Stevenson EC, Turner G, Heaney LG, et al. Bronchoalveolar lavage findings suggest two different forms of childhood asthma. Clin Exp Allergy 1997; 27: 1027-1035.

12. Le Bourgeois M, Goncalves M, Le Clainche L, et al. Bronchoalveolar cells in children $<3$ years old with severe recurrent wheezing. Chest 2002; 122: 791-797.

13. Saglani S, Payne DN, Zhu J, et al. Early detection of airway wall remodeling and eosinophilic inflam mation in preschool wheezers. Am J Respir Crit Care Med 2007; 176: 858-864.

14. Payne DN, Wilson NM, James A, et al. Evidence for different subgroups of difficult asthma in children. Thorax 2001; 56: 345-350.
15. Payne D, Bush A. Phenotype-specific treatment of difficult asthma in children. Paediatr Respir Rev 2004; 5: 116-123.

16. Fleming L, Tsartsali L, Wilson N, et al. Sputum inflammatory phenotypes are not stable in children with asthma. Thorax 2012; 67: 675-681.

17. McLean WH, Palmer CN, Henderson J, et al. Filaggrin variants confer susceptibility to asthma. J Allergy Clin Immunol 2008; 121: 1294-1295.

18. Lazic N, Roberts G, Custovic A, et al. Multiple atopy phenotypes and their associations with asthma: similar findings from two birth cohorts. Allergy 2013; 68: 764-770.

19. Garden FL, Simpson JM, Marks GB, et al. Atopy phenotypes in the Childhood Asthma Prevention Study (CAPS) cohort and the relationship with allergic disease: clinical mechanisms in allergic disease. Clin Exp Allergy 2013; 43: 633-641.

20. Moffatt MF, Gut IG, Demenais F, et al. A large-scale, consortium-based genomewide association study of asthma. N Engl J Med 2010; 363: 1211-1221.

21. Granell R, Henderson AJ, Timpson N, et al. Examination of the relationship between variation at 17921 and childhood wheeze phenotypes. J Allergy Clin Immunol 2013; 131: 685-694.

22. Spycher BD, Henderson I, Granell R, et al. Genomewide prediction of childhood asthma and related phenotypes in a longitudinal birth cohort. J Allergy Clin Immunol 2012; 130: 503-509.

23. Ferreira MA, Matheson MC, Tang CS, et al. Genomewide association analysis identifies 11 risk variants associated with the asthma with hay fever phenotype. J Allergy Clin Immunol 2013 [In press, DOI: 10.1016/ j.jaci.2013.10.030].

24. Melen E, Granell R, Kogevinas M, et al. Genome-wide association study of body mass index in 23000 individuals with and without asthma. Clin Exp Allergy 2013; 43: 463-474.

25. van der Valk RJ, Duijts L, Timpson NJ, et al. Fraction of exhaled nitric oxide values in childhood are associated with 17q11.2-q12 and 17q12-921 variants. J Allergy Clin Immunol 2013 [In press, DOI: 10.1016/ j.jaci.2013.08.053]

26. Repapi E, Sayers I, Wain LV, et al. Genome-wide association study identifies five loci associated with lung function. Nat Genet 2010; 42: 36-44.

27. Bonnelykke K, Matheson MC, Pers TH, et al. Metaanalysis of genome-wide association studies identifies ten loci influencing allergic sensitization. Nat Genet 2013; 45: 902-906. 